EXTENDED REPORT

\title{
Blue on yellow perimetry with scanning laser ophthalmoscopy in patients with age related macular disease
}

\author{
A Remky, A E Elsner
}

Br J Ophthalmol 2005;89:464-469. doi: 10.1136/bjo.2004.050260

\begin{abstract}
See end of article for authors' affiliations

....................

Correspondence to: Dr A Remky, Augenklinik der Med Fak der RWTH Aachen, Pauwelsstrasse 30, 52057 Aachen, Germany; andreas.remky@ post.rwth-aachen.de
\end{abstract}

Accepted for publication 1 September 2004 length sensitive (SWS) cone mechanism sensitivity is related to Background/aim: The loss of short wavelength sensitive (SWS) cone mechanism sensitivity is related to
severe vision loss in patients with age related maculopathy (ARM). A case-control study of patients with ARM and age matched controls was performed, using blue on yellow static perimetry.

Methods: A bright yellow background at $594 \mathrm{~nm}$ isolated the responses of short wavelength cone mechanisms to $458 \mathrm{~nm}$ targets. A scanning laser ophthalmoscope produced stimuli and provided real time, simultaneous fundus illumination. The macula was probed with 16 Goldmann IV targets, 110 degrees from fixation, using a staircase method.

Results: 24 patients with non-exudative ARM were matched to 24 subjects with normal fundus appearance. SWS cone pathway sensitivity for macular targets was significantly reduced in the patients with ARM compared to normals - 15.45 (SD 4.56) dB v $17.22(0.28) \mathrm{dB}$, respectively $(p<0.0005)$. There was not only a diffuse loss of sensitivity in ARM patients, but also a localised loss of sensitivity over drusen $(p<0.025)$. Neither the mean age, $69(8)$ years, nor the mean visual acuity differed between groups, $\log$ MAR $0.09(0.10) v 0.05(0.06)$ for ARM patients $v$ normals, respectively. Patients with soft drusen had lower sensitivity than those with hard drusen $(p<0.05)$.

Conclusion: A loss of SWS cone pathway sensitivity occurred in most patients with early ARM, despite good visual acuity, demonstrating a loss of visual function that cannot be attributed to ageing changes. The loss of sensitivity, despite good visual acuity, included both a diffuse loss and localised losses.
A ge related macular degeneration (AMD) is a major cause of visual impairment in industrialised countries. Funduscopic risk factors for severe vision loss include hyperpigmentation and drusen size, type, and confluency. ${ }^{2}$ Measurements of visual function reflect the physiological status of an individual's retina and illustrate current pathophysiological status rather than past morphological changes. Visual acuity does not reflect retinal health, or cone function, in many diseases. ${ }^{4-12}$ Techniques of measuring visual function are more specific to the locus of pathology of AMD and, less influenced than is visual acuity or contrast sensitivity by lens and other media changes, have generated interest. ${ }^{414-11}$ 13-21

In patients with ARM, short wavelength sensitive cone (SWS) mechanism sensitivity is decreased. ${ }^{12} 1822$ There is decreased sensitivity for short wavelength targets in both the fovea and periphery which is related to poor visual outcome. ${ }^{1423}$ We investigated the SWS cone mechanisms in a case-control design, comparing patients with ARM and good visual acuity to age matched normal subjects. We expanded on the foveal studies by Eisner et al, which minimise the influence of lens and other visual system mechanisms, by testing the posterior pole with blue on yellow perimetry using a scanning laser ophthalmoscope (SLO). ${ }^{24}$

The principle of blue on yellow perimetry is the isolation of the SWS cone response by suppressing responses of other cone types by a yellow background. ${ }^{2}{ }^{12-14} 17-1921$ SWS cone pathways seem more affected by the early stages of various diseases than the other cone pathways. ${ }^{12}{ }^{17} 25$ Fundus perimetry techniques have been developed to visualise the posterior pole and retinal location of the targets. ${ }^{26}$ These methods were improved with the SLO, ${ }^{27}{ }^{28}$ which allowed simultaneous fundus imaging with invisible light and testing with a different laser source. ${ }^{29}$ Correction for eye movements is done by landmark driven adjustment of stimulus presentation $^{30}$ or by correction of the stimulus position after target presentation. ${ }^{31}$

The advantages of fundus perimetry are the identification and quantification of scotomata in patients with unstable or eccentric fixations ${ }^{30}{ }^{32}$ and the assessment of fixation stability. ${ }^{33-35}$ This is particularly valuable in patients with atrophy, exudation, or scars in advanced stages of a variety of diseases. ${ }^{36}{ }^{37}$ However, few studies concentrate on the early changes of diseases or on processes without atrophy and scarring. ${ }^{23} 3138$

\section{MATERIALS AND METHODS}

A research scanning laser ophthalmoscope was used. ${ }^{39}$ The technique of blue on yellow perimetry has been described previously. ${ }^{24}{ }^{40}$ Perimetry was performed using two different lasers. Blue stimuli (200 ms) and a blue background $(29 \times 23$ degrees, $93 \mathrm{td})$ were generated by a multiline argon laser at $458 \mathrm{~nm}$ and a six cavity interference filter. A yellow background $(4100 \mathrm{td})$, produced by a $594 \mathrm{~nm}$ HeNe laser, was superimposed. Real time, simultaneous fundus illumination was provided by the Cr:Li:SaF laser with a Gaussian beam profile at $850 \mathrm{~nm}$, which was invisible to the subject and provided excellent image quality. SWS cones have their peak sensitivities at low spatial frequencies, and thus large rectangular targets $(0.9 \times 0.9$ degrees $0.24 \times 0.24 \mathrm{~mm}$ that approximate Goldmann IV and a large fixation cross $(2.3 \times 2.3$ degrees $)$ at maximum intensity were used.

Abbreviations: $A M D$, age related macular degeneration; ARM, age related maculopathy; RPE, retinal pigment epithelium; SLO, scanning laser ophthalmoscopy; SWS, short wavelength sensitive; VA, visual acuity 
The eye with the better visual acuity, or if no difference in visual acuity the right eye, was tested. All patients and subjects received mydriatics before retinal examination.

Static perimetry was performed using software previously described. ${ }^{24}{ }^{31}$ Stimuli were presented in random order in a static, single crossing staircase strategy similar to that in Fastpac. ${ }^{41}$ The answer could be ignored by the examiner in case of an inappropriate response-for example, at the beginning of the test, in case of large eye movements, or during misalignment of the eye with respect to the apparatus.

Sixteen stimulus loci in a rectangular grid were tested in a 10 degree central field. About 100 test stimuli were presented, taking about 10-15 minutes. Additional infrared imaging was performed to determine fundus status using large and small field sizes, $28.6 \times 23.0$ degrees and $15.2 \times 13.6$ degrees, and both direct and indirect modes. ${ }^{42} 43$

\section{Data analysis}

Sensitivity is defined as the target seen with the lowest intensity in $d B(1 / 10 \log$ unit). Maximum sensitivity was defined as $20 \mathrm{~dB}$. The range of usable stimulus intensity was 2.0-2.5 log units (maximal intensity - background intensity). An attenuation of $20 \mathrm{~dB}$ indicated excellent sensitivity, while $10 \mathrm{~dB}$ indicated poor sensitivity.

To compute the retinal location of the target, a fundus landmark was chosen. A retinal image was digitised $17 \mathrm{~ms}$ after stimulus offset. On each image, the examiner placed a cursor on the landmark to compute the corrected retinal location of the stimulus. A confocal, infrared image was digitised at the end of the test, on which the correct locations of stimuli were displayed.

To determine whether a patient had sensitivity reduced on average, rather than increased variability, Bebié functions were computed ${ }^{44}$ Each subject's data were sorted by rank order, then plotted with the leftmost point being the most sensitive. A displacement downward of the Bebié function
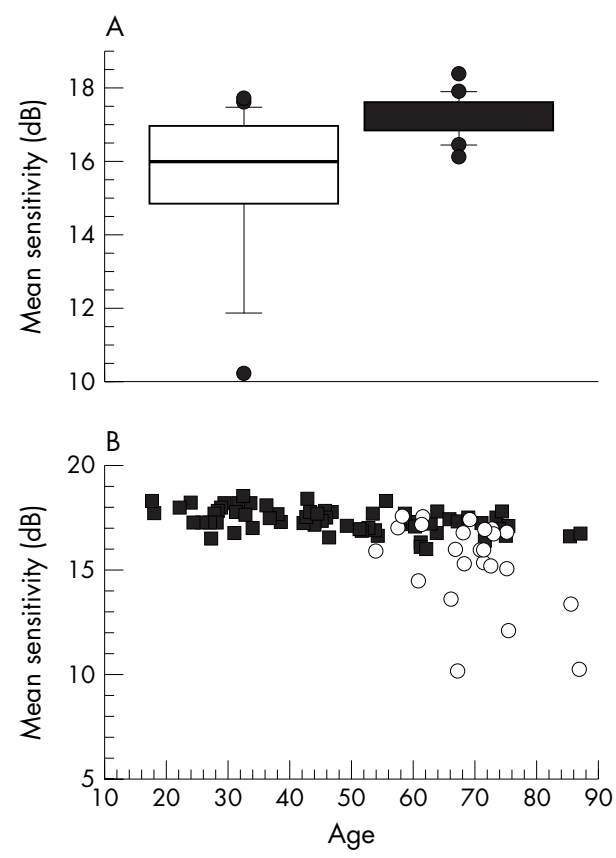

Figure 1 Sensitivity of blue targets on yellow backgrounds for normals $\checkmark$ patients with ARM, showing worse performance, as well as more variable, for patients on average. (A) Sensitivity for patients and subjects, matched for age, shown as box plots with 10, 25, 50, and 75 percentiles. (B) Sensitivity as a function of age for all normals from Remky et al $^{24}$ compared with patients. indicates diffuse loss, while a negative slope indicates large variability among loci.

Mean and standard deviation was computed for the sensitivity for each group, as defined below. The unpaired $t$ test was used to assess the difference in sensitivity, visual acuity, and age. To compare sensitivity over areas of drusen, nine patients were selected with clearcut regions of drusen versus no drusen, from the fundus slide. The mean sensitivity for each region was computed. Then the mean sensitivity for the same stimulus loci was computed for matched controls. A new variable was formed, the difference between drusen versus no drusen areas, for each subject. An unpaired $t$ test was performed on these differences. A $t$ test was also performed on the difference between "normal" areas in patient eyes and the same loci in control eyes.

\section{Subjects and patients}

The ARM group consisted of 24 patients. To investigate early changes of ARM, the inclusion criteria were no atrophic region $>200 \mu \mathrm{m}$, no exudative lesions, and visual acuity $\leqslant 20 / 40$. Patients were examined by a retinal specialist. Snellen visual acuity was assessed before examination. For statistical analysis, all acuity scores were converted to logarithmic equivalents (log maximal angle of resolution, $\log$ MAR). Fundus photography was performed, and fluorescein angiography as needed for clinical purposes. Drusen within the central 15 degree visual angle were graded using colour fundus slides. Eyes were assigned to one of two categories by drusen type predominance, hard or soft, according to the international classification. ${ }^{45}$ One patient with multiple hard drusen that had softened and become confluent was placed into the predominantly soft group. One patient exhibited whitish fundus lesions with a peau d'orange pattern, fitted into neither group, and was excluded from subgroup analysis. The control group consisted of 24 normal subjects, selected from the previous study ${ }^{24}$ to match patients for age and sex, had no history of eye disease in the test eye.

Before participation in the study, all subjects received a detailed explanation of the procedures and gave their informed consent and signed a consent form approved by the institutional review board of the Schepens Eye Research Institute. The tenets of the Declaration of Helsinki were followed.

\section{RESULTS}

Blue on yellow perimetry showed that the mean sensitivity of ARM patients was significantly lower compared with controls $(p<0.005)$, despite no difference in age or visual acuity $(\mathrm{p}=0.87$ and $\mathrm{p}=0.08$, respectively) (figlA, table 1$)$. Patients had higher inter-individual variation than controls: coefficients of variation $14 \% \vee 3 \%(\mathrm{p}<0.0005)$. Sensitivity in the maculas of patients ranged from normal to an average decrease of more than $7 \mathrm{~dB}$ (fig 1B). Patients had no consistent pattern of field loss such as that found previously in ageing-for example, central $v$ more peripheral locations or nasal $v$ inferior field, although a difference $-0.23 \mathrm{~dB}$ for the

Table 1 Comparison of patients with age related maculopathy (ARM) to controls for sensitivity of blue on yellow perimetry, age, visual acuity in logMAR, and sex

\begin{tabular}{lll}
\hline & ARM patients & Controls \\
\hline Sensitivity (dB) & $15.45(4.56)$ & $17.22(0.28)$ \\
Age (years) & $68.9(7.9)$ & $68.6(7.9)$ \\
LogMAR & $-0.089(0.098)$ & $-0.048(0.055)$ \\
Sex (M/F) & $10 / 14$ & $9 / 15$ \\
\hline
\end{tabular}




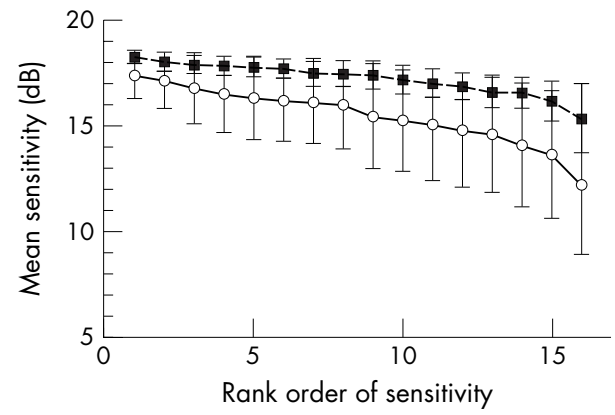

Figure 2 Comparison of sensitivity between matched normal subjects and patients, plotted as means and standard deviations. The Bebié functions emphasise the difference between groups in overall sensitivity or diffuse loss, shown by the relative heights of the curves connecting the loci. The greater differences among loci is shown by the slope, with normals having a function with nearly zero slope, indicating little difference among loci, but patients having on average a negative slope, but with large individual differences. Solid lines, patients. Broken line, normals.

central versus more peripheral locations was observed in the total sample $(\mathrm{p}<0.05)$.

The hard drusen group had 13 eyes, and the soft drusen group had 10 eyes. The mean sensitivity of patients with hard drusen was significantly higher than those with soft, 16.52 $($ SD 0.88$) \mathrm{dB} v 14.31(2.72) \mathrm{dB}$, respectively $(\mathrm{p}<0.05)$. The soft drusen group had significantly greater intraindividual variability across loci than the hard drusen group: coefficients of variation $15 \% v 6.6 \%$, respectively $(\mathrm{p}<0.005)$. There was no difference in age or visual acuity between groups.

To investigate whether loss of mean sensitivity is caused by localised morphological changes or diffuse loss, we assessed
Bebie curves. The grand average for the patients with ARM was shifted to a lower sensitivity value, indicating a diffuse field loss (fig 2). Slopes were steeper for patients than for normals, indicating the influence of localised changes $\mathrm{p}<0.0005)$. Figures $3-5$ illustrate the differences in sensitivity and slope for individual Bebié functions. The curve for patients with soft drusen was steeper than for those with hard drusen $(p<0.005)$, indicating more variation of sensitivity over the field. The slope of the Bebie function was significantly steeper than the normal slope for each patient shown.

In patients with ARM, sensitivity was typically low over areas with fundus changes such as drusen, confluent material, atrophic patches, or hyperpigmentation (figs 3-5). The low sensitivity over areas of pathology corresponded to the increased variability of sensitivity across the field for patients, particularly those with soft drusen (table 2). Retinal areas with drusen had lower sensitivity than areas lacking drusen on the fundus slide $(p<0.025)$, as previously reported. ${ }^{38}$ Retinal areas without visible soft drusen in the eyes of patients with ARM also had worse sensitivity than the matched control loci $(p<0.01)$. This, again, indicates a diffuse loss of sensitivity in the group of patients.

Often the pathological changes visible ophthalmoscopically or in the fundus slide did not adequately represent the extent of changes, the severity of changes, the amount of hyperpigmentation, or the confluency of drusen that was seen on infrared imaging (figs 3C, 3D, 4C). The confocal mode of infrared imaging (figs 3B, 4B, and 5B) showed many more drusen and most features more distinctly. The indirect mode of infrared imaging readily distinguished atrophic from other lightly pigmented or highly reflective features (fig 4C), as well as regions of confluent material from pale regions. These images indicate that even the more normal appearing
A

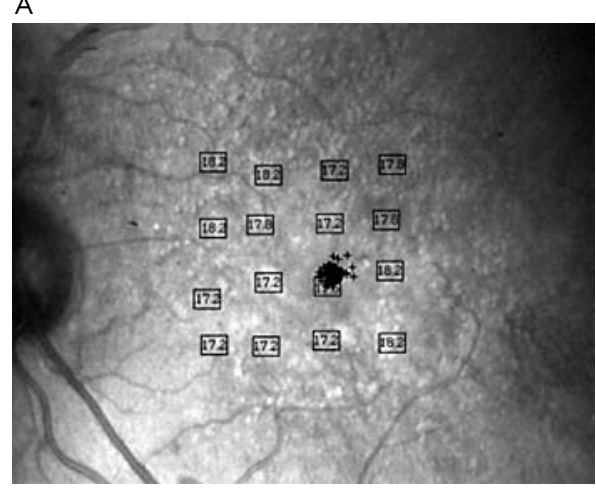

C
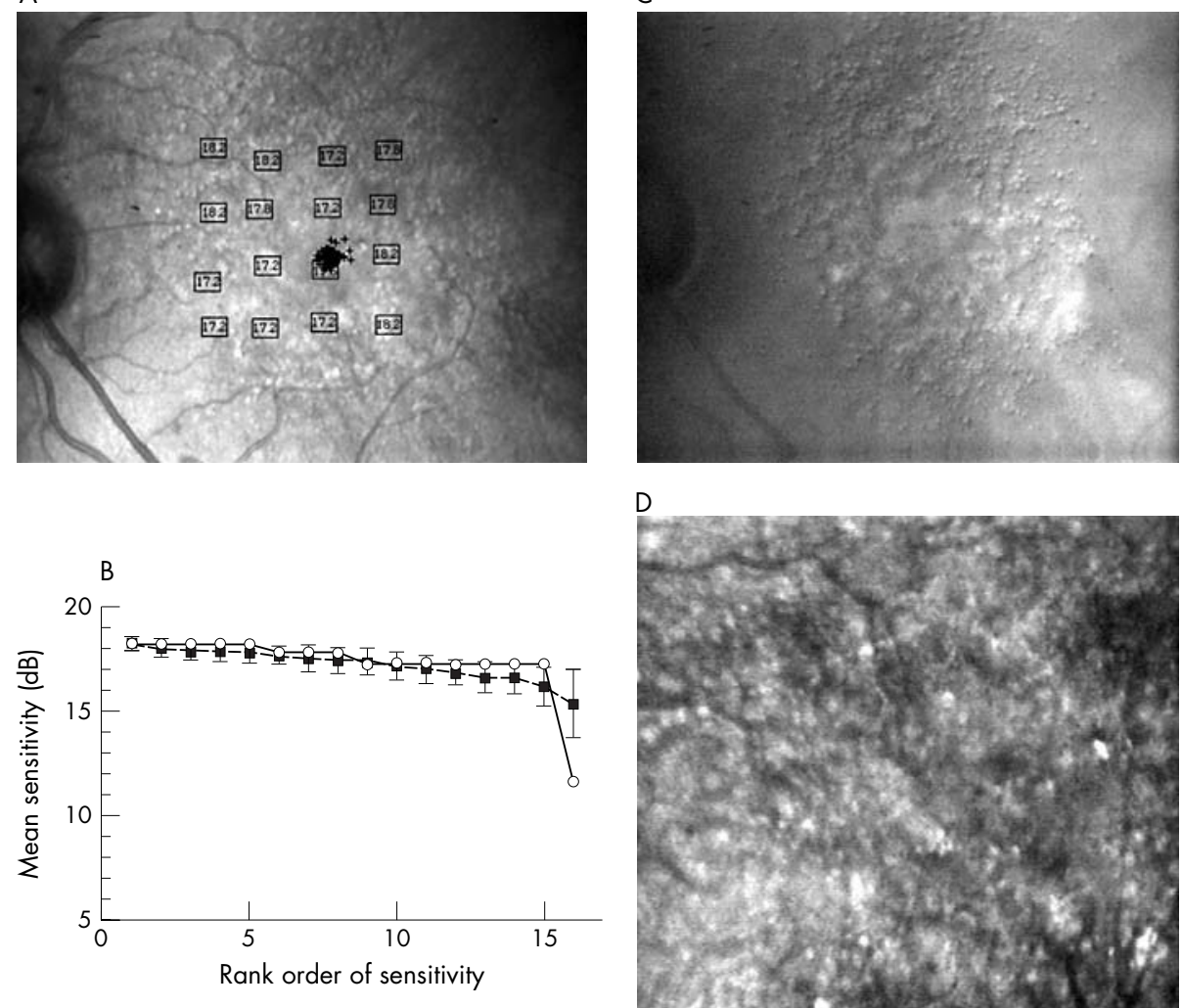

D

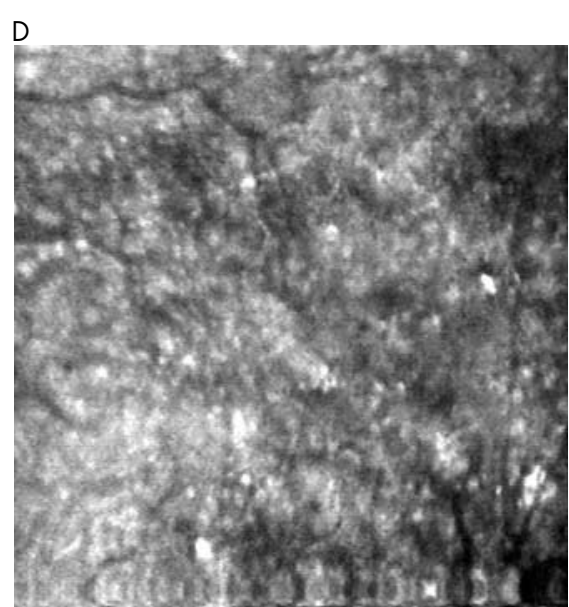

Figure 3 Blue on yellow perimetry results for a 67 year old male patient with visual acuity (VA) 20/25, good fixation, and multiple hard drusen that soffened and became confluent. (A) Results in dB, plotted on the final infrared confocal image. (B) Bebié function with open symbols for the patient data and closed symbols for the average of control subjects. The patient's function has a slope of -0.14 , showing similar performance between the patient and the controls, but with one locus significantly worse for the patient, $11.6 \mathrm{~dB}$. This locus is nearest the fixation cross and in the centre of the confluent material $(C, D)$, near clumped hyperpigmentation. (C) Indirect mode infrared image, large field size, showing multiple hard drusen surrounding a thickened area of confluent material, and not atrophy. (D) Indirect mode infrared image, small field size, showing magnified view of drusen, the confluent material, and areas of hyperpigmentation in addition to those seen ophthalmoscopically. 
A
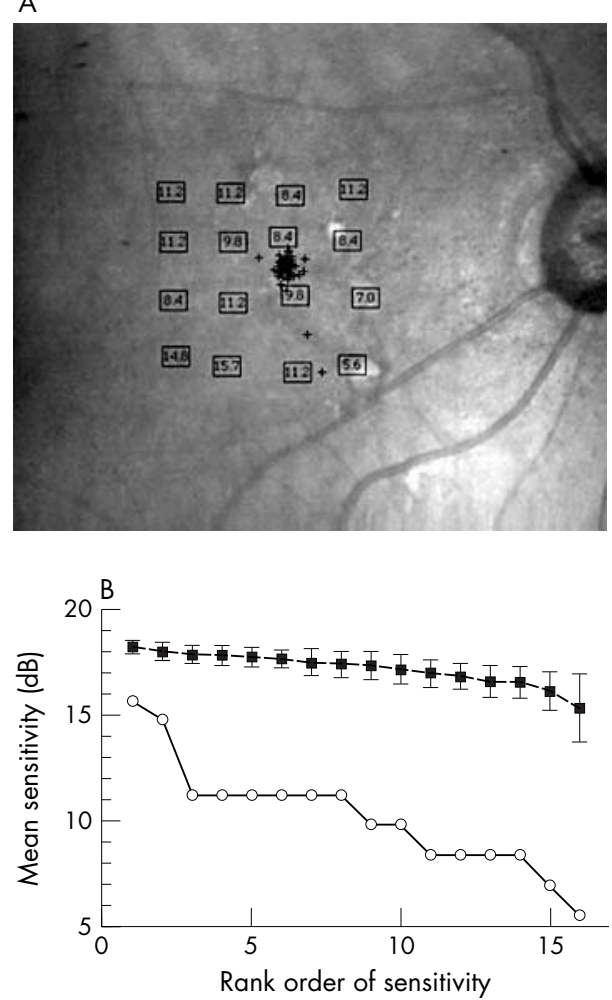

C

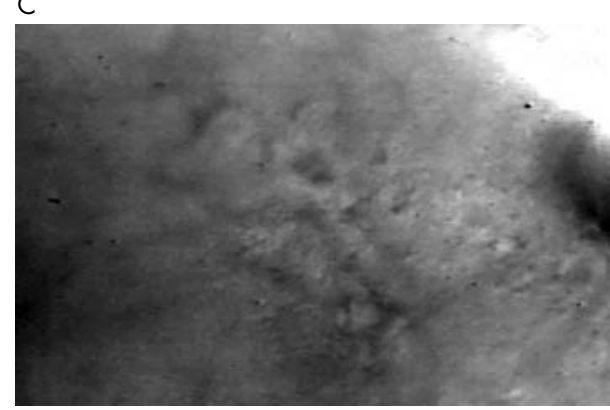

Figure 4 Blue on yellow perimetry results for an 86 year old female patient with visual acuity (VA) 20/25, good fixation, and distinct soft drusen. (A) Results in $d B$, plotted on the final confocal infrared image. This is the only patient with atrophic areas. One is beneath the bottom, rightmost stimulus loci. The other is in the top row, between two loci. The worst sensitivity for this patient was at the atrophic site. Other loci with poor sensitivity, near the optic nerve head, fell onto regions of confluent material and hyperpigmentation (see C). (B) Bebié function for the patient $v$ the average of controls' data, as in figure 3B, showing most loci had worse sensitivity than the controls' data, as well as a greater difference among loci in sensitivity as seen from the slope of -0.051 . (C) Indirect mode infrared image showing multiple soft drusen in a $25 \times 19$ degree area. There is a region of image saturation over the optic nerve head. There is a thickened area of confluent material near the optic nerve head, not to be mistaken for atrophy but containing clumped hyperpigmentation.

areas of the posterior pole may have involvement in disease, and that some pathological features may not be adequately documented by fundus slides. Thus, a sensitivity loss that is diffuse and widespread is not surprising in light of the infrared imaging findings.

\section{DISCUSSION}

SWS cone sensitivity, reported to be more affected by the early stages of several diseases than the other cone pathways, was investigated in a parametric study of the macula. The
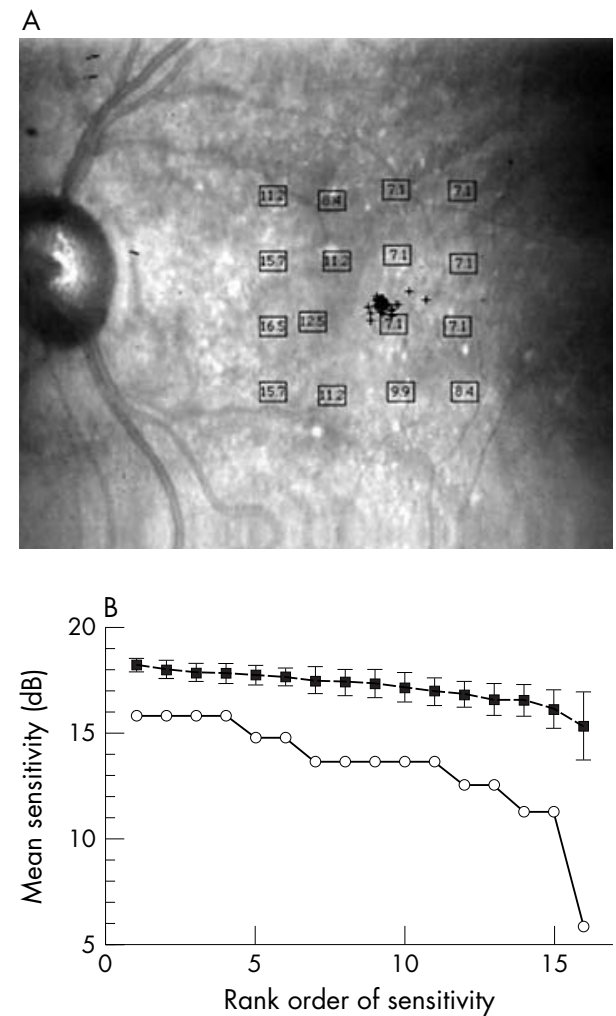

Figure 5 Blue on yellow perimetry results for an 85 year old male patient with visual acuity (VA) $20 / 25$, good fixation, and confluent soft drusen. (A) Results in $\mathrm{dB}$, plotted on the final confocal infrared image. (B) Bebie function as in figure $3 B$ with a slope of 0.031 , showing that most loci had worse sensitivity, as well as a somewhat greater difference among loci in sensitivity.

loss of SWS cone pathway sensitivity in patients with ARM found in this study agrees with other studies. ${ }^{2} 12141746$ This loss was not attributable to ageing alone, since a strict matching procedure was performed.

Previous studies have shown early functional abnormalities-for example, decreased retinal sensitivity for short wavelength stimuli under dark adapted sensitivity, ${ }^{46}$ and decreased SWS cone sensitivity ${ }^{2}{ }^{14}$ in patients with drusen and hyperpigmentation. The change of SWS sensitivity over time correlates well with changes of fundus appearance. Changes of the RPE (retinal pigment epithelium), particularly drusen and hyperpigmentation, are the fundus changes that are visible early in ARM. ${ }^{47}$ These are related to pathophysiological processes of the RPE. The site of sensitivity loss may reflect pathological changes to the photoreceptors, when these metabolically active cells receive reduced levels of oxygen and other metabolites or accumulate waste products locally. Evidence of drusen, particular confluent drusen, may indicate local or diffuse dysfunction of the retinal pigment epithelium (RPE), as well as physical disruption of the tissues. Our finding of more pronounced sensitivity losses in the group with soft drusen is consistent with the clinico-histopathological hypotheses of greater loss of metabolic or hydrophilic barrier function.

There are changes found for the pathways not only for the SWS but also for the LWS and MWS cones, demonstrated with a variety of methods ranging from those that isolate cone photopigment properties to those that rely on cone inputs for the detection of flicker and spatial patterns. ${ }^{810111448}$ The relative degree of damage to the cone pathways depend upon not only the severity of damage to the 
Table 2 Comparison of blue on yellow sensitivity over areas with as opposed to without drusen

\begin{tabular}{llllll}
\hline & ARM patients & & \multicolumn{2}{c}{ Controls } & \\
\cline { 2 - 3 } \cline { 5 - 5 } & Loci over drusen & Non-drusen area & & $\begin{array}{l}\text { Corresponding loci } \\
\text { for drusen area }\end{array}$ & $\begin{array}{l}\text { Corresponding loci } \\
\text { for non-drusen area }\end{array}$ \\
\hline Sensitivity $(\mathrm{dB})$ & $13.11(3.12)$ & $15.20(2.24)$ & $16.77(0.51)$ & $17.22(0.30)$ \\
\hline
\end{tabular}

RPE complex, but also the type of test used and the scaling of the data. ${ }^{49}$

An early, selective loss of SWS mechanism sensitivity has also been found in glaucoma, diabetes, and other retinopathies..$^{13} 171950$ The primary site of pathophysiology in these diseases is the cells of the inner retina, which are susceptible to ischaemic and other insults. Since the SWS mechanism pathways lack the redundancy that other cone pathways have, either a photoreceptor/RPE complex or inner retinal change may cause loss of function at a specific retinal locus. This may improve the sensitivity of the test, but worsen the specificity of detecting the tissue locus of damage.

The present data show not only a significant diffuse loss of SWS sensitivity in ARM, but also a reduction of sensitivity caused by localised fundus changes, such as drusen. This is in contrast with the studies with dark adapted thresholds, ${ }^{46}$ in which no differences in sensitivity over drusen were found compared to neighbouring areas, regardless of type of drusen. If sensitivity loss has a basis in inner retinal ischaemia, then only certain types of losses are consistent with our perimetry findings. Significant, localised changes to the circulation supporting the nerve fibre layer are inconsistent with our data, since sensitivity loss does not extend over large retinal areas, such as arcuate or step patterns. However, more widespread and less severe changes to the inner retina could be related to our diffuse loss findings. Previous studies provide predictive information about exudation and severe vision loss, although not differences in short wavelength loss at specific loci. ${ }^{23}$ While exudation has been hypothesised to depend upon factors that may cause diffuse loss, such as ischaemia, $^{5152}$ it remains to be determined whether the diffuse loss or localised losses are more predictive for severe visual loss.

SWS cone pathway function testing is a quick, noninvasive procedure that can easily be performed at the posterior pole in patients with ARM. Using an SLO provides the advantages of minimising lens and small pupil effects, and providing fundus imaging for comparison. Using automatic, static perimetry allows the testing of different locations without the fundus appearance leading to experimenter bias. Our data displayed sensitivity losses beyond ageing changes. The prognostic value has been reported to be high for previous studies of the SWS cone pathway in patients with ARM. ${ }^{14}$ Our finding of large individual differences among patients implies that despite all patients having good visual acuity, some eyes already have much worse function and may be at risk for severe vision loss from geographic atrophy ${ }^{48}$ and exudation. ${ }^{53}$

\section{ACKNOWLEDGEMENTS}

The authors thank Dr CL Trempe and A Morandi for best assistance in recruiting and examining the patients and for helpful discussions.

\section{Authors' affiliations}

A Remky, A E Elsner, Schepens Eye Research Institute, Harvard Medical School, 20 Staniford Street, Boston, MA 02114, USA

A Remky, Augenklinik der Med Fak der RWTH Aachen, Pauwelsstrasse 30, 52057 Aachen, Germany
This investigation was supported by $\operatorname{Re} 805 / 2-1$ (Deutsche Forschungsgemeinschaft, Bonn, Germany) to AR and EY007624 (Bethesda, MD) to AE.

\section{REFERENCES}

1 Leibowitz H, Kruger DE, Maunder LR, et al. The Framingham Eye study Monograph: an ophthalmological and epidemiological study of cataract, glaucoma, diabetic retinopathy, macular degeneration and visual acuity in a general population of 2631 adults, 1973-1975. Surv Ophthalmol 1980;24(Suppl):335-610.

2 Eisner A, Stoumbos VD, Klein ML, et al. Relations between fundus appearance and function. Invest Ophthalmol Vis Sci 1991;32:8-20.

3 Smiddy WE, Fine SL: Prognosis of patients with bilateral macular drusen. Ophthalmology 1984;91:271.

4 Burns SA, Elsner AE, Lobes LAJ. Foveal cone photopigment bleaching in central serous retinopathy. Applied Optics 1988;27:1045-9.

5 Elsner AE, Burns SA, Beausencourt E, et al. Foveal cone photopigment distribution: small alterations associated with macular pigment distribution. Invest Ophthalmol Vis Sci 1998;39:2394-404.

6 Elsner AE, Burns SA, Lobes LA Jr. Foveal cone pigment optical density in retinitis pigmentosa. Applied Optics 1987;26:1378-84.

7 Elsner AE, Burns SA, Lobes LAJ, et al. Photopigment bleaching abnormalities in diabetes. Invest Ophthalmol Vis Sci 1987;28:718-23.

8 Elsner AE, Burns SA, Weiter JJ. Cone photopigment in older subjects: decreased optical density in ealy age-related macular disease. J Opt Soc Am A 2002; 19:215-22.

9 Elsner AE, Moraes L, Kunze CW, et al. Foveal cone photopigment distribution in age-related macular degeneration: association with fundus features. Vision Science and Its Applications Technical digest series 1998;1:14-17.

10 Mayer MJ, Spiegler SJ, Ward B, et al. Mid-frequency loss of foveal flicker sensitivity in early stages of age-related maculopathy. Invest Ophthalmol Vis Sci 1992:33:3136-42.

11 Mayer MJ, Ward B, Klein R, et al. Flicker sensitivity and fundus appearance in pre-exudative age related maculopathy. Invest Ophthalmol Vis Sci 1994;35: 1138-49.

12 Applegate RA, Adams AJ, Cavender JC, et al. Early color vision changes in age-related maculopathy. Applied Optics 1987;26:1458-62.

13 Crognale MA, Rabin J, Switkes E, et al. Selective loss of S-pathway sensitivity in central serous choroidopathy revealed by spatio-chromatic visual evoked cortical potential (VECP). In: Drum B, ed. Colour vision deficiencies XI. Amsterdam: Kluwer Academic Publishers, 1993:229-39.

14 Eisner A, Klein ML, Zilis JD, et al. Visual function and the subsequent development of exudative age-related macular degeneration. Invest Ophthalmol Vis Sci 1992;33:3091-102.

15 Falsini B, Fadda A, larossi $G$, et al. Retinal sensitivity to flicker modulation: reduced by early age-related maculopathy. Invest Ophthalmol Vis Sci 2000;41:1498-506.

16 Falsini B, Serrao S, Fadda A, et al. Focal electroretinograms and fundus appearance in nonexudative age-related macular degeneration. Quantitative relationship between retinal morphology and function. Graefes Arch Clin Exp Ophthalmol 1999;237:193-200.

17 Greenstein VC, Hood DC, Ritch R, et al. S (blue) cone pathway vulnerability in retinitis pigmentosa, diabetes and glaucoma. Invest Ophthalmol Vis Sci 1989;30: 1732-7.

18 Haegerstrom-Portnoy G, Brown B: Two-color increment threshold in early age-related maculopathy. Clin Vis Sci 1989;4:165-72.

19 Johnson CA, Adams AJ, Casson EJ, et al. Blue-on-yellow perimetry can predict the development of glaucomatous visual field loss. Arch Ophthalmol 1993:111:645-50.

20 Li J, Tso MOM, Lam TT. Reduced amplitude and delayed latency in foveal response of multifocal electroretinogram in early age related degeneration. $\mathrm{Br} J$ Ophthalmol 2001;85:287-90.

21 Marré M, Marré E. Erworbene Störungen des Farbensehens: Diagnostik. Stuttgart: Gustav Fischer Verlag, 1986.

22 Remky A, Lichtenberg K, Elsner AE, et al. Short-wavelength automated perimetry in age-related maculopathy. Br J Ophthalmol 2001;85:1432-26.

23 Sunness JS, Johnson MA, Massof RW, et al. Retinal sensitivity over drusen and nondrusen areas. Arch Ophthalmol 1988;106:1081.

24 Remky A, Elsner AE, Morandi A, et al. Blue-on-yellow perimetry with a SLO: Small Alterations in the Central Macula with Aging. J Opt Soc Am A $2001 ; 18: 1425-36$.

25 Remky A, Arend O, Hendricks S. Short-wavelength automated perimetry and capillary density in early diabetic maculopathy. Invest Ophthalmol Vis Sci 2000;41:274-82

26 Sunness JS, Johnson MA, Massof RW, et al. Wilmer fundus camera stimulator. Applied Optics 1987;26:1487-91. 
27 Elsner AE, Timberlake GT, Burns SA, et al. High illuminance perimetry: photopigment mechanisms. Noninvasive assessment of the Visual System. OSA Annual Meeting, 1989 Technical digest series 1989:XX.

28 Timberlake GT, Mainster MA, Peli E, et al. Reading with a macular scotoma I. Retinal location of scotoma and fixation area. Invest Ophthalmol Vis Sci 1986;27:1137-47.

29 Chen J-F, Elsner AE, Burns SA, et al. The effect of eye shape on retinal responses. Clin Vis Sci 1992;7:521-30.

30 Sunness JS, Schuchard RA, Shen N, et al. Landmark-driven fundus perimetry using the scanning laser ophthalmoscope. Invest Ophthalmol Vis Sci 1995;36:1863-74.

31 Toonen F, Remky A, Janssen V, et al. Microperimetry in patients with central serous retinopathy. Ger J Ophthalmol 1995:4:311-4.

32 Fletcher DC, Schuchard RA, Livingstone CL, et al. Scanning laser ophthalmoscope macular perimetry and applications for low vision rehabilitation clinicians. Ophthalmol Clin N Am 1994:7:257-265.

33 Rohrschneider K, Becker M, Kruse FE, et al. Stability of fixation: results of fundus-controlled examination using the scanning laser ophthalmoscope. German J Ophthalmol 1995;4:197-202.

34 Schuchard RA, Fletcher DC, JM. A scanning laser ophthalmoscope (SLO) lowvision rehabilitation system. Clin Eye Vis Care 1994;6:101-7.

35 Schuchard RA, Raasch TW: Retinal locus for fixation: pericentral fixation targets. Clin Vis Sci 1993;7:511-20.

36 Loewenstein A, Sunness JS, Bressler NM, et al. Scanning laser ophthalmoscope fundus perimetry after surgery for choroidal neovascularization. Am J Ophthalmol 1998;125:657-65.

37 Rohrschneider K, Glück R, Becker M, et al. Scanning laser fundus perimetry before laser photocoagulation of well defined choroidal neovascularisation. Br J Ophthalmol 1997;81:568-73.

38 Takamine $Y$, Shiraki K, Moriwaki M, et al. Retinal sensitivity measurements over drusen using scanning laser ophthalmoscope microperimetry. Graefes Arch Clin Exp Ophthalmol 1998;236:285-90.

39 Elsner AE, Burns SA, Hughes GW, et al. Reflectometry with a scanning laser ophthalmoscope. Appl Optics 1992;31:3697-710.

40 Remky A, Beausencourt E, Elsner AE. Angioscotometry with the SLO: Comparison of the effect of different wavelength. Invest Ophthalmol Vis Sci 1996;37:2350-5.
41 Flanagan JG, Moss ID, Wild JM, et al. Evaluation of Fastpac: a new strategy for threshold estimation with the Humphrey Field analyser. Graefes Arch Clin Exp Ophthalmol 1993;231:465-9.

42 Elsner AE, Burns SA, Weiter JJ, et al. Infrared imaging of sub-retinal structures in the human ocular fundus. Vis Res 1996:36:191-205.

43 Hartnett ME, Elsner AE. Characteristics of exudative age-related macular degeneration determined in vivo with confocal and indirect infrared imaging Ophthalmology 1996;103:58-71.

44 Funkhouser AT, Fankhauser F, Weale R. Problems related to diffuse versus localized loss in the perimetry of glaucomatous visual fields. Graefes Arch Clin Exp Ophthalmol 1992;230:243-7.

45 The International ARM Epidemiological Study Group. An international classification and grading system for age-related maculopathy and agerelated macular degeneration. Surv Ophthalmol 1995:39:367-74.

46 Sunness JS, Massof RW, Johnson MA, et al. Diminished foveal sensitivity may predict the development of advanced age-related macular degeneration. Ophthalmology 1989;96:357-81.

47 Sarks SH. Drusen and their relationship to senile macular degeneration. Aust J Ophthalmol 1980:89:117-30.

48 Sunness JS, Rubin GS, Applegate CA, et al. Visual function abnormalities and prognosis in eyes with age-related geographic atrophy of the macula and good visual acuity. Ophthalmology 1997; 104:1677-91.

49 McKeefry DJ, Parry NR, Murray IJ. Simple reaction times in color space: the influence of chromaticity, contrast, and cone opponency. Invest Ophthalmol Vis Sci 2003;44:2267-76.

50 Sample PA, Weinreb RN. Color perimetry for assessment of primary openangle glaucoma. Invest Ophthalmol Vis Sci 1990;31:1869-75.

51 Hartnett ME, Weiter JJ, Staurenghi G, et al. Deep retinal vasuclar anomalous complexes in advanced age-related macular degeneration. Ophthalmology 1996; 103:2042-53.

52 Kuhn D, Meunier O, Soubrane G, et al. Imaging of chorioretinal anastomoses in vascularized retinal pigment epithelium detachments. Arch Ophthalmol 1995:113:1392-8.

53 Macular Photocoagulation Study Group. Risk factors for choroidal neovascularization in the second eye of patients with juxtafoveal or subfoveal choroidal neovascularization secondary to age-related macular degeneration. Arch Ophthalmol 1997;115:741-7. 